\title{
COMBINATORIAL EXAMPLES IN UNIVERSAL COALGEBRA. III
}

\author{
ROBERT C. DAVIS
}

\begin{abstract}
Categories cotripleable over the category of sets, for which the right adjoint at 2 has six elements and the terminal object more than one element, are surveyed along with some related categories. Some are related to multigraphlike structures, and others to sets acted upon by monoids.
\end{abstract}

Introduction. A natural way to follow up the results reported in [2] would be to search for cotripleable categories defined by a single operator for which $|F(2)|=6$. At first sight, this seems hopeless, since the total number of possible structures on a six-element set is $64^{64}$, nearly four quadrillion googol. However, this total is very quickly and drastically reduced by applying various symmetries, eliminating notational variants and equivalent structures, and exploiting the existence and uniqueness of maps induced by the fact that $F$ is a right adjoint.

This search seems best organized on the basis of the size of $T=F(1)$, the terminal object. For $|T|=3$, it follows easily from $F(2)=T+T$ that each structure $A$ is uniquely determined by the map $A \rightarrow T$, and we have only the category of sets over 3. For $|T|=2$, there are the uninteresting examples consisting of disjoint unions of an unstructured set and an object from one of the types II through VII in [2]. A long search has uncovered only four nontrivial cases, whose implications are discussed in this paper. The situation for $|T|=1$ will be treated in a later article.

1. Categories related to multigraphs. Three of our four examples are similar to the bizarre, unwieldy types IV-VII in [2]. The structures on $F(2)$ are easily described. Let $F(2)=\{0,1,2,3,4,5\}$, with adjunction $0,1,2 \rightarrow 0$ and $3,4,5 \rightarrow 1$. For each subset $E$ of $F(2), \omega(E)$ contains 0 and 3, and also contains nothing else except:

In Case $1, \omega(\{0,1,2\})=\omega(\{1,2,3\})=\{0,2,3\}$ and $\omega(\{0,4,5\})=\omega(\{3,4,5\})=$ $\{0,3,5\}$;

In Case 2, when $E$ contains exactly one of 2,5 (which is in $\omega(E)$ ), exactly one of 1,4 and exactly one of 0,3 ;

In Case 3, when $E$ contains 2 and/or 5 (which is/are in $\omega(E)$ ), exactly one of 1,4 and exactly one of 0,3 .

In these cases, no description seems available except that the objects are multigraph-like structures, with "connecting sequences" that are homomorphic images of $F(2)$, and maps that are locally homomorphisms on the connecting sequences.

Received by the editors November 14, 1983.

1980 Mathematics Subject Classification. Primary 18C15; Secondary 08C05.

Key words and phrases. Cotripleable category, right adjoint, Beck condition.

(C)1984 American Mathematical Society $0002-9939 / 84 \$ 1.00+\$ .25$ per page 
2. Categories related to monoid actions. The remaining example is more interesting. Let $M$ be the monoid $\{e, m\}$ with $m^{2}=m$. The objects of the category are simply $M$-sets; the maps are those ordinary homomorphisms that satisfy the additional requirement for $f: A \rightarrow B$ that $F(a)=m b$ implies $a=m c$ for some $c$ in $A$. The same definition makes sense for any monoid in place of $M$, but the proof of the Beck condition for cotripleability only works for this specific $M$ and does not generalize (except of course that the situation is trivial if the monoid is a group). The right adjoint is given by $F(A)=A \cup(A \times A)$ (disjoint union), with $m a=a$ and $m(a, b)=a$, so we indeed have $|T|=2$ and $|F(2)|=6$.

An analogue of sorts can be found for a few other monoids. For example, if $N=\left\{e, m, m^{2}\right\}$ with $m^{3}=m^{2}$, consider the category of $N$-sets $A$ partitioned into $A_{1}, A_{2}, A_{3}$, and $A_{4}$, where $A_{1}=m^{2} A, A_{4}=\left\{a \mid m a \neq m^{2} a\right\}$, and $m A_{4} \subseteq A_{3}$. A map is an ordinary homomorphism satisfying $f\left(A_{i}\right) \subseteq B_{i}$. This is weaker than the condition in the above paragraph, since $m A_{4}$ is not required to equal $A_{3}$, but it does satisfy the Beck criterion. The right adjoint is similar, $F(A)=A \cup(A \times A) \cup(A \times$ $A) \cup(A \times A \times A)$ with $m(a, b, c)=(a, b)$. This construction does not seem to generalize to any quasicotripleable category for more general monoids.

This unsatisfactory situation changes if we strengthen the requirement for a map over a general monoid $N$ to read:

(*) for all $m$ in $N, f(a)=m b$ implies $a=m c$ for some $c$ such that $f(c)=b$. This condition is less unnatural than appears at first. The general description of quasicotripleable categories in [1] leads us to consider categories definable in terms of operators $\omega_{A}: 2^{A} \rightarrow 2^{A}$ and homomorphisms $f: A \rightarrow B$ with $\omega_{A} f^{-1}(E)=f^{-1} \omega_{B}(E)$ for all subsets $E$ of $B$. One might at first think that the ordinary category of $N$-sets would arise if one defines for each $m$ an operator $\omega(E)=m E$, but instead one obtains the category of $N$-sets and maps satisfying (*). (For ordinary homomorphisms without this requirement, one needs $\omega(E)=m^{-1} E$.) For every $N$, this category is quasicotripleable. In at least some cases it is in fact cotripleable, i.e. there is a right adjoint, but even for very simple monoids the right adjoint is fairly complex. For example, with the $M$ considered earlier, $m^{2}=m, F(A)$ is constructed as follows. For each pair ( $E, a)$ where $E$ is a subset of $A$ and $a \in A$, for each $e$ in $E$ we put a point in the $e$ th compartment and let $m$ send all these points to another point in the $a$ th compartment. If $B$ is an $M$-set and $g: B \rightarrow A$ is any function, the unique map $f: B \rightarrow F(A)$ induced by it sends each $b$ in $m B$, together with the points in $m^{-1} b$, onto the points of $F(A)$ supplied for the pair $\left(g\left(\left(m^{-1} b\right)-a\right), g(a)\right)$. If $A$ has $n$ elements, in constructing $F(A)$, for each choice of an $r$-element subset and a basepoint we include $r+1$ elements. Thus, the total number of points in $F(A)$ is

$$
n\left(\left(\begin{array}{l}
n \\
0
\end{array}\right)+2\left(\begin{array}{l}
n \\
1
\end{array}\right)+3\left(\begin{array}{l}
n \\
2
\end{array}\right)+\cdots+(n+1)\left(\begin{array}{l}
n \\
n
\end{array}\right)\right)=2^{n-1} n(n+2) .
$$

In particular, $|T|=3$ and $|F(2)|=16$. Just as in the case of the category of $M$-sets and open homomorphisms (which has $|F(2)|=12$, not 6 as was stated in [2]), similar constructions work for any finite cyclic monoid. But the question of the existence of 
the right adjoint for arbitrary monoids continues to baffle this author, and appears to be one of the most difficult problems in this area.

\section{REFERENCES}

1. R. C. Davis, Quasicotripleable categories, Proc. Amer. Math. Soc. 32 (1972), 43-48.

2. ___ Combinatorial examples in universal coalgebra. II, Proc. Amer. Math. Soc. 89 (1983), 35-38.

Department of Mathematics, Southern Methodist University, Dallas, Texas 75275 